\title{
Boundedness for Riesz transform associated with Schrödinger operators and its commutator on weighted Morrey spaces related to certain nonnegative potentials
}

\section{Yu Liu* and Lijuan Wang}

"Correspondence:

liuyu75@pku.org.cn

School of Mathematics and Physics,

University of Science and

Technology Beijing, Beijing, 100083, China

\begin{abstract}
Let $L=-\Delta+V$ be a Schrödinger operator, where $\Delta$ is the Laplacian on $\mathbb{R}^{n}$ and the nonnegative potential $V$ belongs to the reverse Hölder class $B_{q}$ for $q \geq n / 2$. The Riesz transform associated with the operator $L$ is denoted by $T=\nabla(-\Delta+V)^{-\frac{1}{2}}$ and the dual Riesz transform is denoted by $T^{*}=(-\Delta+V)^{-\frac{1}{2}} \nabla$. In this paper, we establish the boundedness for the operator $T^{*}$ and its commutator on the weighted Morrey spaces $L_{\alpha \vee \omega}^{p, \lambda}\left(\mathbb{R}^{n}\right)$ related to certain nonnegative potentials belonging to the reverse Hölder class $B_{q}$ for $n / 2 \leq q<n$, where $p_{0}^{\prime}<p<\infty$ and $\frac{1}{p_{0}}=\frac{1}{q}-\frac{1}{n}$.
\end{abstract}

Keywords: Morrey spaces; commutator; reverse Hölder class; Schrödinger operator; Riesz transform

\section{Introduction}

In this paper, we consider the Schrödinger operator

$$
L=-\Delta+V(x) \text { on } \mathbb{R}^{n}, n \geq 3,
$$

where $V(x)$ is a nonnegative potential belonging to the reverse Hölder class $B_{q}$ for $q \geq n / 2$. The Riesz transform associated with the Schrödinger operator $L$ is defined by $T=\nabla L^{-\frac{1}{2}}$ and the commutator operator

$$
[b, T](f)(x)=T(b f)(x)-b(x) T f(x), \quad x \in \mathbb{R}^{n},
$$

where $f$ is a suitable integral function. Also, the dual Riesz transform associated with the Schrödinger operator $L$ is defined by $T^{*}=L^{-\frac{1}{2}} \nabla$ and the commutator operator

$$
\left[b, T^{*}\right](f)(x)=T^{*}(b f)(x)-b(x) T^{*} f(x), \quad x \in \mathbb{R}^{n} .
$$

First, Tang and Dong established the boundedness of some Schrödinger type operators on the Morrey spaces related to the nonnegative potential $V$ belonging to the reverse Hölder class in [1]. Furthermore, Liu and Wang investigated the boundedness of the dual

\section{Springer}

C2014 Liu and Wang; licensee Springer. This is an Open Access article distributed under the terms of the Creative Commons Attribution License (http://creativecommons.org/licenses/by/2.0), which permits unrestricted use, distribution, and reproduction in any medium, provided the original work is properly cited. 
Riesz transforms and its commutators on the Morrey spaces related to the nonnegative potential $V$ belonging to the reverse Hölder class in [2]. Recently, Pan and Tang established the boundedness of some Schrödinger type operators on weighted Morrey spaces related to the nonnegative potential $V$ belonging to the reverse Hölder class in [3]. Motivated by [3], our aim is to establish the boundedness for the dual Riesz transform associated with Schrödinger operators and its commutators on weighted Morrey spaces related to the certain nonnegative potentials, where the condition on the potential is weaker than that in [3]. Our result is a nontrivial generalization of the main results in [3].

A nonnegative locally $L^{q}$ integrable function $V(x)$ on $\mathbb{R}^{n}$ is said to belong to $B_{q}(1<q<$ $\infty$ ) if there exists $C>0$ such that the reverse Hölder inequality

$$
\left(\frac{1}{|B|} \int_{B} V(x)^{q} d x\right)^{1 / q} \leq C\left(\frac{1}{|B|} \int_{B} V(x) d x\right)
$$

holds for every ball $B$ in $\mathbb{R}^{n}$.

It is important that the $B_{q}$ class has a property of 'self improvement'; that is, if $V \in B_{q}$, then $V \in B_{q+\varepsilon}$ for some $\varepsilon>0$ (see [4]).

We assume the potential $V \in B_{q}$ for $q \geq n / 2$ throughout the paper. We introduce the auxiliary function $\rho(x, V)=\rho(x)$ defined by

$$
\rho(x)=\sup _{r>0}\left\{r: \frac{1}{r^{n-2}} \int_{B(x, r)} V(y) d y \leq 1\right\}, \quad x \in \mathbb{R}^{n} .
$$

It is well known that $0<\rho(x)<\infty$ for any $x \in \mathbb{R}^{n}$ (cf. Lemma 1 in Section 2).

A kind of new Morrey spaces is established by Tang and Dong in [1]. Furthermore, the weighted Morrey space is introduced by Pan and Tang in [3]. Let $p \in[1, \infty), \alpha \in(-\infty, \infty)$, and $\lambda \in[0,1)$. For $f \in L_{\text {loc }}^{p}\left(\mathbb{R}^{n}\right)$ and $V \in B_{q}(q>1)$, we say $f \in L_{\alpha, V, \omega}^{p, \lambda}\left(\mathbb{R}^{n}\right)$ (weighted Morrey spaces related to the nonnegative potential $V$ ) provided that

$$
\|f\|_{L_{\alpha, V, \omega}^{p, \lambda}\left(\mathbb{R}^{n)}\right.}^{p}=\sup _{B\left(x_{0}, r\right) \subset \mathbb{R}^{n}}\left(1+\frac{r}{\rho\left(x_{0}\right)}\right)^{\alpha} \omega\left(B\left(x_{0}, 2 r\right)\right)^{-\lambda} \int_{B\left(x_{0}, r\right)}|f(x)|^{p} \omega(x) d x<\infty,
$$

where $B=B\left(x_{0}, r\right)$ denotes a ball with centered at $x_{0}$ and radius $r$, and the weight functions $\omega \in A_{p}^{\rho, \infty}$ (see Section 2).

Now we are in a position to give the main results in this paper.

Theorem 1 Suppose $V \in B_{q}$ for $n / 2 \leq q<n, \alpha \in(-\infty, \infty), \lambda \in(0,1)$, and $1 / p_{0}=1 / q-1 / n$. Then, for $p_{0}^{\prime} \leq p<\infty$ and $\omega \in A_{p / p_{0}^{\prime}}^{\rho, \infty}$,

$$
\left\|T^{*} f\right\|_{L_{\alpha, V, \omega}^{p, \lambda}\left(\mathbb{R}^{n}\right)} \leq C\|f\|_{L_{\alpha, V, \omega}^{p, \lambda}\left(\mathbb{R}^{n}\right)^{\prime}}
$$

where $C$ is independent off.

Theorem 2 Suppose $V \in B_{q}$ for $n / 2 \leq q<n, b \in B M O_{\rho}, \alpha \in(-\infty, \infty), \lambda \in(0,1)$, and $p_{0}$ so that $1 / p_{0}=1 / q-1 / n$. Then, for $p_{0}^{\prime} \leq p<\infty$ and $\omega \in A_{p / p_{0}^{\prime}}^{\rho, \infty}$,

$$
\left\|\left[b, T^{*}\right] f\right\|_{L_{\alpha, V, \omega}^{p, \lambda}\left(\mathbb{R}^{n}\right)} \leq C\|f\|_{L_{\alpha, V, \omega}^{p, \lambda}\left(\mathbb{R}^{n}\right)},
$$

where $C$ is independent off . 
We will use $C$ to denote a positive constant, which is not necessarily same at each occurrence and even is different in the same line, and may depend on the dimension $n$ and the constant in (3). By $A \sim B$, we mean that there exists a constant $C$ such that $1 / C \leq A / B \leq C$.

\section{Some lemmas}

In this section, we collect some known results proved in [4] in order to prove the main results in this paper.

Lemma 1 There exist constants $C, k_{0}>0$ such that

$$
\frac{1}{C}\left(1+\frac{|x-y|}{\rho(x)}\right)^{-k_{0}} \leq \frac{\rho(y)}{\rho(x)} \leq C\left(1+\frac{|x-y|}{\rho(x)}\right)^{k_{0} /\left(k_{0}+1\right)} .
$$

In particular, $\rho(y) \sim \rho(x)$ if $|x-y|<C \rho(x)$.

Lemma 2 (1) For $0<r<R<\infty$,

$$
\frac{1}{r^{n-2}} \int_{B(x, r)} V(y) d y \leq C\left(\frac{r}{R}\right)^{2-\frac{n}{q}} \frac{1}{R^{n-2}} \int_{B(x, R)} V(y) d y
$$

and

$$
\frac{1}{r^{n-2}} \int_{B(x, r)} V(y) d y \sim 1 \quad \text { if and only if } \quad r \sim \rho(x) .
$$

(2) There exist $C>0$ and $l_{0}>0$ such that

$$
\frac{1}{R^{n-2}} \int_{B(x, R)} V(y) d y \leq C\left(1+\frac{R}{\rho(x)}\right)^{l_{0}} .
$$

Let $\mathcal{K}$ be the kernel of $T$ and $\mathcal{K}^{*}$ be the kernel of $T^{*}$.

Lemma 3 If $V \in B_{q}$ for $q \geq n / 2$, then for every $N$ there exists a constant $C_{N}>0$ such that

$$
\left|\mathcal{K}^{*}(x, z)\right| \leq \frac{C_{N}}{\left(1+\frac{|x-z|}{\rho(x)}\right)^{N}} \frac{1}{|x-z|^{n-1}}\left(\int_{B(z,|x-z| / 4)} \frac{V(u)}{|u-z|^{n-1}} d u+\frac{1}{|x-z|}\right) .
$$

Moreover, the last inequality also holds with $\rho(x)$ replaced by $\rho(z)$.

In this paper, we always write $\Psi_{\theta}(B)=\left(1+r / \rho\left(x_{0}\right)\right)^{\theta}$, where $\theta>0 ; x_{0}$ and $r$ denote the center and radius of $B$, respectively.

A weight will always mean a nonnegative function which is locally integrable. As in [5], we say that a weight $\omega$ belongs to the class $A_{p}^{\rho, \theta}$ for $1<p<\infty$, if there is a positive constant $C$ such that for the whole ball $B=B(x, r)$

$$
\left(\frac{1}{\Psi_{\theta}(B)|B|} \int_{B} \omega(y) d y\right)\left(\frac{1}{\Psi_{\theta}(B)|B|} \int_{B} \omega^{-\frac{1}{p-1}}(y) d y\right)^{p-1} \leq C .
$$

We also say that a nonnegative function $\omega$ satisfies the $A_{1}^{\rho, \theta}$ condition if there exists a positive constant $C$, for all balls $B$

$$
M_{V}^{\theta}(\omega)(x) \leq C \omega(x), \quad \text { a.e. } x \in \mathbb{R}^{n},
$$


where

$$
M_{V}^{\theta} f(x)=\sup _{x \in B} \frac{1}{\Psi_{\theta}(B)|B|} \int_{B}|f(y)| d y .
$$

Since $\Psi_{\theta}(B) \geq 1$, obviously, $A_{p} \subset A_{p}^{\rho, \theta}$ for $1 \leq p<\infty$, where $A_{p}$ denote the classical Muckenhoupt weights (see [6]). It follows from [7] that $A_{p} \subset \subset A_{p}^{\rho, \theta}$ for $1 \leq p<\infty$. For convenience, we always assume that $\Psi(B)$ denotes $\Psi_{\theta}(B), A_{p}^{\rho, \infty}=\bigcup_{\theta>0} A_{p}^{\rho, \theta}$, and $A_{\infty}^{\rho, \infty}=$ $\bigcup_{p \geq 1} A_{p}^{\rho, \infty}$.

Lemma 4 ([7]) Let $0<\theta<\infty$, then:

(i) If $1 \leq p_{1}<p_{2}<\infty$, then $A_{p_{1}}^{\rho, \theta} \subset A_{p_{2}}^{\rho, \theta}$.

(ii) $\omega \in A_{p}^{\rho, \theta}$ if and only if $\omega^{-\frac{1}{p-1}} \in A_{p^{\prime}}^{\rho, \theta}$, where $1 / p+1 / p^{\prime}=1$.

(iii) If $\omega \in A_{p}^{\rho, \theta}$ for $1 \leq p<\infty$, then there exists a constant $C>0$ such that for any $\lambda>1$

$$
\omega\left(\lambda B\left(x_{0}, r\right)\right) \leq C\left(1+\frac{\lambda r}{\rho\left(x_{0}\right)}\right)^{\left(k_{0}+1\right) \theta} \omega\left(B\left(x_{0}, r\right)\right)
$$

Lemma 5 ([8]) Let $0<\theta<\infty, 1 \leq p<\infty$. If $\omega \in A_{p}^{\rho, \theta}$, then there exist positive constants $\delta$, $\eta$, and $C$ such that

$$
\left(\frac{1}{|B|} \int_{B} \omega(y)^{1+\delta} d y\right)^{1 /(1+\delta)} \leq C \frac{1}{|B|} \int_{B} \omega(y) d y\left(1+\frac{r}{\rho\left(x_{0}\right)}\right)^{\eta}
$$

for all ball $B\left(x_{0}, r\right)$.

As a consequence of Lemma 5, we have the following result.

Corollary 1 ([8]) Let $0<\theta<\infty, 1 \leq p<\infty$. If $\omega \in A_{p}^{\rho, \theta}$, then there exist positive constants $q>1, \eta$, and $C$ such that

$$
\frac{\omega(E)}{\omega(B)} \leq C\left(\frac{|E|}{|B|}\right)^{1 / q}\left(1+\frac{r}{\rho\left(x_{0}\right)}\right)^{\eta}
$$

for any measurable subset $E$ of a ball $B\left(x_{0}, r\right)$.

Bongioanni et al. [9] introduced a new space $B M O_{\theta}(\rho)$ defined by

$$
\|f\|_{B M O_{\theta}(\rho)}=\sup _{B \subset \mathbb{R}^{n}} \frac{1}{\Psi_{\theta}(B)|B|} \int_{B}\left|f(x)-f_{B}\right| d x<\infty,
$$

where $f_{B}=\frac{1}{|B|} \int_{B} f(y) d y$ and $\Psi_{\theta}(B)=\left(1+r / \rho\left(x_{0}\right)\right)^{\theta}, B=B\left(x_{0}, r\right)$, and $\theta>0$.

In particularly, Bongioanni et al. [9] proved the following results for $B M O_{\theta}(\rho)$.

Proposition 1 Let $\theta>0$ and $1 \leq s<\infty$. If $b \in B M O_{\theta}(\rho)$, then

$$
\left(\frac{1}{|B|} \int_{B}\left|b(x)-b_{B}\right|^{s} d x\right)^{1 / s} \leq C\|b\|_{B M O_{\theta}(\rho)}\left(1+\frac{r}{\rho\left(x_{0}\right)}\right)^{\theta^{\prime}}
$$

holds for all $B=B\left(x_{0}, r\right)$, with $x_{0} \in \mathbb{R}^{n}$ and $r>0$, where $\theta^{\prime}=\left(k_{0}+1\right) \theta$ and $k_{0}$ is the constant appearing in Lemma 1. 
Proposition 2 Let $b \in B M O_{\theta}(\rho), B=B\left(x_{0}, r\right)$, and $1 \leq s<\infty$. Then

$$
\left(\frac{1}{\left|2^{k} B\right|} \int_{2^{k} B}\left|b(y)-b_{B}\right|^{s} d y\right)^{\frac{1}{s}} \leq C\|b\|_{B M O_{\theta}(\rho)} k\left(1+\frac{2^{k} r}{\rho\left(x_{0}\right)}\right)^{\theta^{\prime}}
$$

for all $k \in \mathbb{N}$, with $\theta^{\prime}=\left(k_{0}+1\right) \theta$ and the constant $k_{0}$ is given as in Proposition 1 .

Obviously, the classical $B M O$ space is properly contained in $B M O_{\theta}(\rho)$; for more examples please see [9]. For convenience, we let $B M O_{\rho}=\bigcup_{\theta>0} B M O_{\theta}(\rho)$.

From Corollary 2.2 in [3], the following result holds true.

Corollary 2 If $b \in B M O_{\rho}$ and $\omega \in A_{\infty}^{\rho, \infty}$, then there exist positive constants $C$ and $\eta$ such that for every ball $B=B(x, r)$, we have

$$
\frac{1}{\omega(B)} \int_{B}\left|b(x)-b_{B}\right|^{p} \omega(x) d x \leq\left(1+\frac{r}{\rho(x)}\right)^{\eta}\|b\|_{B M O_{\rho}}^{p}
$$

where $b_{B}=\frac{1}{|B|} \int_{B} b(y) d y$.

\section{The proof of our main results}

Proof of Theorem 1 Without loss of generality, we may assume that $\alpha<0$ and $\omega \in A_{p / p_{0}^{\prime}}^{\rho, \theta}$. Pick any ball $B=B\left(x_{0}, r\right)$, and write

$$
f(x)=f_{1}(x)+f_{2}(x)
$$

where $f_{1}=\chi_{B\left(x_{0}, 2 r\right)} f$. Hence, we have

$$
\begin{aligned}
& \left(\int_{B\left(x_{0}, r\right)}\left|T^{*} f(x)\right|^{p} \omega(x) d x\right)^{1 / p} \\
& \quad \leq\left(\int_{B\left(x_{0}, r\right)}\left|T^{*} f_{1}(x)\right|^{p} \omega(x) d x\right)^{1 / p}+\left(\int_{B\left(x_{0}, r\right)}\left|T^{*} f_{2}(x)\right|^{p} \omega(x) d x\right)^{1 / p} .
\end{aligned}
$$

By the $L_{\omega}^{p}$ boundedness of $T^{*}$ (see Theorem 3 in [5]), we obtain

$$
\int_{B\left(x_{0}, r\right)}\left|T^{*} f_{1}(x)\right|^{p} \omega(x) d x \leq C\left(1+\frac{r}{\rho\left(x_{0}\right)}\right)^{-\alpha} \omega\left(B\left(x_{0}, 2 r\right)\right)^{\lambda}\|f\|_{L_{\alpha, V, \omega}^{p, \lambda}}^{p}\left(\mathbb{R}^{n}\right)
$$

Now, for $x \in B\left(x_{0}, r\right)$ and using Lemma 3, we have

$$
\begin{aligned}
\left|T^{*} f_{2}(x)\right| & =\left|\int_{\left|x_{0}-z\right|>2 r} \mathcal{K}^{*}(x, z) f(z) d z\right| \\
& \leq I_{1}(x)+I_{2}(x)
\end{aligned}
$$

where

$$
I_{1}(x)=C_{N} \int_{\left|x_{0}-z\right|>2 r} \frac{|f(z)|}{|x-z|^{n}\left(1+\frac{|x-z|}{\rho(x)}\right)^{N}} d z
$$


and

$$
I_{2}(x)=C_{N} \int_{\left|x_{0}-z\right|>2 r} \frac{|f(z)|}{|x-z|^{n-1}\left(1+\frac{|x-z|}{\rho(x)}\right)^{N}} \int_{B(z,|x-z| / 4)} \frac{V(u)}{|u-z|^{n-1}} d u d z .
$$

Then

$$
\begin{aligned}
& \left(\int_{B\left(x_{0}, r\right)}\left|T^{*} f_{2}(x)\right|^{p} \omega(x) d x\right)^{1 / p} \\
& \quad \leq\left(\int_{B\left(x_{0}, r\right)}\left(I_{1}(x)\right)^{p} \omega(x) d x\right)^{1 / p}+\left(\int_{B\left(x_{0}, r\right)}\left(I_{2}(x)\right)^{p} \omega(x) d x\right)^{1 / p} .
\end{aligned}
$$

By the proof of Theorem 1.1 in [3], we have

$$
\int_{B\left(x_{0}, r\right)}\left(I_{1}(x)\right)^{p} \omega(x) d x \leq C\left(1+\frac{r}{\rho\left(x_{0}\right)}\right)^{-\alpha} \omega\left(B\left(x_{0}, 2 r\right)\right)^{\lambda}\|f\|_{L_{\alpha, V, \omega}^{p, \lambda}\left(\mathbb{R}^{n}\right)}^{p} .
$$

Next we deal with $I_{2}(x)$. For $x \in B\left(x_{0}, r\right), \frac{\left|x_{0}-z\right|}{2} \leq|x-z| \leq \frac{3\left|x_{0}-z\right|}{2}$. We get

$$
\begin{aligned}
\int_{B\left(x_{0}, r\right)} & \left(I_{2}(x)\right)^{p} \omega(x) d x \\
= & C_{N} \int_{B\left(x_{0}, r\right)}\left(\int_{\left|x_{0}-z\right|>2 r} \frac{|f(z)|}{|x-z|^{n-1}\left(1+\frac{|x-z|}{\rho(x)}\right)^{N}} \int_{B(z,|x-z| / 4)} \frac{V(u)}{|u-z|^{n-1}} d u d z\right)^{p} \omega(x) d x \\
\leq & \sum_{i=1}^{\infty} C_{N} \int_{B\left(x_{0}, r\right)}\left(\int_{B\left(x_{0}, 2^{i+1} r\right) \backslash B\left(x_{0}, 2^{i} r\right)} \frac{|f(z)|}{\left|x_{0}-z\right|^{n-1}\left(1+\frac{\left|x_{0}-z\right|}{\rho(x)}\right)^{N}}\right. \\
& \left.\times \int_{B\left(x_{0}, 2^{i+3} r\right)} \frac{V(u)}{|u-z|^{n-1}} d u d z\right)^{p} \omega(x) d x \\
\leq & \sum_{i=1}^{\infty} C_{N} \int_{B\left(x_{0}, r\right)} \frac{1}{\left(1+\frac{2^{i} r}{\rho(x)}\right)^{N p}}\left(2^{i} r\right)^{-(n-1) p}\left(\int_{B\left(x_{0}, 2^{i} r\right)}|f(z)| \mathcal{I}_{1}\left(V \chi_{B\left(x_{0}, 2^{i} r\right)}\right) d z\right)^{p} \omega(x) d x .
\end{aligned}
$$

Let $p_{0}^{\prime} \leq p<\infty$. By simple computation, $\frac{p}{p_{0}^{\prime}}=1+\frac{p}{v}$. By the definition of $A_{p / p_{0}^{\prime}}^{\rho, \theta}$,

$$
\begin{aligned}
& \left(\frac{1}{\Psi\left(B\left(x_{0}, 2^{i} r\right)\right)\left|B\left(x_{0}, 2^{i} r\right)\right|} \int_{B\left(x_{0}, 2^{i} r\right)} \omega^{-v / p}(y) d y\right)^{1 / v} \\
& \quad=\left(\frac{1}{\Psi\left(B\left(x_{0}, 2^{i} r\right)\right)\left|B\left(x_{0}, 2^{i} r\right)\right|} \int_{B\left(x_{0}, 2^{i} r\right)} \omega^{-\frac{1}{\frac{p}{v}+1-1}}(y) d y\right)^{\left(\frac{p}{v}+1-1\right) \frac{1}{v\left(\frac{p}{v}+1-1\right)}} \\
& \quad \leq C\left(\frac{1}{\Psi\left(B\left(x_{0}, 2^{i} r\right)\right)\left|B\left(x_{0}, 2^{i} r\right)\right|} \int_{B\left(x_{0}, 2^{i} r\right)} \omega(y) d y\right)^{-\frac{1}{p}}
\end{aligned}
$$

where $1 / q=1 / s+1 / n$ and $1 / p+1 / v+1 / s=1$.

Using Hölder's inequality, (10), and the boundedness of the fractional integral $\mathcal{I}_{1}: L^{q} \rightarrow$ $L^{s}$ with $1 / q=1 / s+1 / n$, for $1 / p+1 / v+1 / s=1$, we have

$$
\begin{aligned}
& \int_{B\left(x_{0}, 2^{i} r\right)}|f(x)| \mathcal{I}_{1}\left(V \chi_{B\left(x_{0}, 2^{i} r\right)}\right) d x \\
& \quad=\int_{B\left(x_{0}, 2^{i} r\right)}|f(x)| \omega^{1 / p} \omega^{-1 / p} \mathcal{I}_{1}\left(V \chi_{B\left(x_{0}, 2^{i} r\right)}\right) d x
\end{aligned}
$$




$$
\begin{aligned}
\leq & \left(\Psi\left(B\left(x_{0}, 2^{i} r\right)\right)\left|B\left(x_{0}, 2^{i} r\right)\right|\right)^{\frac{1}{v}}\left(\int_{B\left(x_{0}, 2^{i} r\right)}|f(x)|^{p} \omega(x) d x\right)^{1 / p} \\
& \times\left(\frac{1}{\Psi\left(B\left(x_{0}, 2^{i} r\right)\right)\left|B\left(x_{0}, 2^{i} r\right)\right|} \int_{B\left(x_{0}, 2^{i} r\right)} \omega(x)^{-v / p} d x\right)^{1 / v} \\
& \times\left(\int_{B\left(x_{0}, 2^{i} r\right)}\left(\mathcal{I}_{1}\left(V \chi_{B\left(x_{0}, 2^{i} r\right)}\right)\right)^{s} d x\right)^{1 / s} \\
\leq & C\left(\Psi\left(B\left(x_{0}, 2^{i} r\right)\right)\left|B\left(x_{0}, 2^{i} r\right)\right|\right)^{\frac{1}{v}}\left(\int_{B\left(x_{0}, 2^{i} r\right)}|f(x)|^{p} \omega(x) d x\right)^{1 / p} \\
& \times\left(\frac{1}{\Psi\left(B\left(x_{0}, 2^{i} r\right)\right)\left|B\left(x_{0}, 2^{i} r\right)\right|} \int_{B\left(x_{0}, 2^{i} r\right)} \omega(x) d x\right)^{-1 / p} \\
& \times\left(\int_{B\left(x_{0}, 2^{i} r\right)}\left(\mathcal{I}_{1}\left(V \chi_{B\left(x_{0}, 2^{i} r\right)}\right)\right)^{s} d x\right)^{1 / s} \\
\leq & C\left(\Psi\left(B\left(x_{0}, 2^{i} r\right)\right)\left|B\left(x_{0}, 2^{i} r\right)\right|\right)^{1 / p+1 / v}\left(\int_{B\left(x_{0}, 2^{i} r\right)}|f(x)|^{p} \omega(x) d x\right)^{1 / p} \\
& \times \omega\left(B\left(x_{0}, 2^{i} r\right)\right)^{-1 / p}\left\|\mathcal{I}_{1}\left(V \chi_{B\left(x_{0}, 2^{i} r\right)}\right)\right\|_{s} \\
\leq & C\left(\Psi\left(B\left(x_{0}, 2^{i} r\right)\right)\left|B\left(x_{0}, 2^{i} r\right)\right|\right)^{1 / p+1 / v}\left(\int_{B\left(x_{0}, 2^{i} r\right)}|f(x)|^{p} \omega(x) d x\right)^{1 / p} \\
& \times \omega\left(B\left(x_{0}, 2^{i} r\right)\right)^{-1 / p}\left\|V \chi_{B\left(x_{0}, 2^{i} r\right)}\right\|_{q} .
\end{aligned}
$$

For $V \in B_{q}$, using Lemma 2, we get

$$
\begin{aligned}
\left\|V \chi_{B\left(x_{0}, 2^{i} r\right)}\right\|_{q} & \leq C\left(2^{i} r\right)^{-n / q^{\prime}} \int_{B\left(x_{0}, 2^{i} r\right)} V(x) d x \\
& \leq C\left(2^{i} r\right)^{-n / q^{\prime}+n-2}\left(2^{i} r\right)^{-n+2} \int_{B\left(x_{0}, 2^{i} r\right)} V(x) d x \\
& \leq C\left(2^{i} r\right)^{-n / q^{\prime}+n-2}\left(1+\frac{2^{i} r}{\rho\left(x_{0}\right)}\right)^{l_{0}} .
\end{aligned}
$$

It is easy to check that $-(n-1) p-\frac{p n}{q^{\prime}}+(n-2) p+n+\frac{p n}{v}=0$. Furthermore, using Corollary 1 , we have

$$
\frac{\omega\left(B\left(x_{0}, 2 r\right)\right)}{\omega\left(B\left(x_{0}, 2^{i+1} r\right)\right)} \leq C\left(2^{i}\right)^{-\frac{n}{q}}\left(1+\frac{2^{i} r}{\rho\left(x_{0}\right)}\right)^{\eta}
$$

Therefore, by (13),

$$
\begin{aligned}
& \int_{B\left(x_{0}, r\right)}\left(I_{2}(x)\right)^{p} \omega(x) d x \\
& \quad=C_{N} \int_{B\left(x_{0}, r\right)}\left(\int_{\left|x_{0}-z\right|>2 r} \frac{|f(z)|}{|x-z|^{n-1}\left(1+\frac{|x-z|}{\rho(x)}\right)^{N}} \int_{B(z,|x-z| / 4)} \frac{V(u)}{|u-z|^{n-1}} d u d z\right)^{p} \omega(x) d x \\
& \quad \leq \sum_{i=1}^{\infty} C_{N}\left(2^{i} r\right)^{-(n-1) p-\frac{p n}{q^{\prime}}+(n-2) p+n+\frac{p n}{v}}
\end{aligned}
$$




$$
\begin{aligned}
& \times \int_{B\left(x_{0}, r\right)} \frac{\left(1+\frac{2^{i} r}{\rho\left(x_{0}\right)}\right)^{\left(1+\frac{p}{v}\right) \theta+l_{0} p}}{\omega\left(B\left(x_{0}, 2^{i} r\right)\right)\left(1+\frac{2^{i} r}{\rho(x)}\right)^{N p}} \int_{B\left(x_{0}, 2^{i} r\right)}|f(y)|^{p} \omega(y) d y \omega(x) d x \\
& \leq \sum_{i=1}^{\infty} C_{N} \omega\left(B\left(x_{0}, 2^{i+1} r\right)\right)^{\lambda-1}\|f\|_{L_{\alpha, V, \omega}^{p, \lambda}}^{p}\left(\mathbb{R}^{n}\right) \int_{B\left(x_{0}, r\right)} \frac{\left(1+\frac{2^{i} r}{\rho\left(x_{0}\right)}\right)^{-\alpha+\left(1+\frac{p}{\nu}\right) \theta+l_{0} p}}{\left(1+\frac{2^{i} r}{\rho(x)}\right)^{N p}} \omega(x) d x \\
& \leq \sum_{i=1}^{\infty} C_{N} \omega\left(B\left(x_{0}, 2^{i+1} r\right)\right)^{\lambda-1} \omega\left(B\left(x_{0}, r\right)\right) \frac{\left(1+\frac{2^{i} r}{\rho\left(x_{0}\right)}\right)^{-\alpha+\left(1+\frac{p}{\nu}\right) \theta+l_{0} p}}{\left(1+\frac{2^{i} r}{\rho\left(x_{0}\right)}\right)^{N p /\left(k_{0}+1\right)}}\|f\|_{L_{\alpha, V, \omega}^{p, \lambda}\left(\mathbb{R}^{n}\right)}^{p} \\
& \leq \sum_{i=1}^{\infty} C_{N}\left(\frac{\omega\left(B\left(x_{0}, 2 r\right)\right)}{\omega\left(B\left(x_{0}, 2^{i+1} r\right)\right)}\right)^{1-\lambda} \omega\left(B\left(x_{0}, 2 r\right)\right)^{\lambda} \frac{\left(1+\frac{2^{i_{r}}}{\rho\left(x_{0}\right)}\right)^{-\alpha+\left(1+\frac{p}{\nu}\right) \theta+l_{0} p}}{\left(1+\frac{2^{i} r}{\rho\left(x_{0}\right)}\right)^{N p /\left(k_{0}+1\right)}}\|f\|_{L_{\alpha, V, \omega}^{p, \lambda}}^{p}\left(\mathbb{R}^{n}\right) \\
& \leq \sum_{i=1}^{\infty} C_{N} 2^{-i n(1-\lambda) / q} \omega\left(B\left(x_{0}, 2 r\right)\right)^{\lambda} \frac{\left(1+\frac{2^{i} r}{\rho\left(x_{0}\right)}\right)^{-\alpha+\left(1+\frac{p}{v}\right) \theta+l_{0} p+\eta}}{\left(1+\frac{2^{i} r}{\rho\left(x_{0}\right)}\right)^{N p /\left(k_{0}+1\right)}}\|f\|_{L_{\alpha, V, \omega}^{p, \lambda}}^{p}\left(\mathbb{R}^{n}\right) \\
& \leq C\|f\|_{L_{\alpha, V, \omega}^{p, \lambda}\left(\mathbb{R}^{n}\right)^{\prime}}^{p}
\end{aligned}
$$

where we choose $N$ large enough so that the above series converges.

From (6)-(14), we obtain

$$
\left\|T^{*} f\right\|_{L_{\alpha, V, \omega}^{p, \lambda}\left(\mathbb{R}^{n}\right)} \leq C\|f\|_{L_{\alpha, V, \omega}^{p, \lambda}\left(\mathbb{R}^{n}\right)}
$$

Thus, Theorem 1 is proved.

Proof of Theorem 2 During the proof of Theorem 2, we always denote $\theta^{\prime}=\left(k_{0}+1\right) \theta$. Without loss of generality, we may assume that $\alpha<0, b \in B M O_{\theta}(\rho)$, and $\omega \in A_{p / p_{0}^{\prime}}^{\rho, \theta}$. Pick any ball $B=B\left(x_{0}, r\right)$, and write

$$
f(x)=f_{1}(x)+f_{2}(x)
$$

where $f_{1}=\chi_{B\left(x_{0}, 2 r\right)} f$. Hence, we have

$$
\begin{aligned}
& \left(\int_{B\left(x_{0}, r\right)}\left|\left[b, T^{*}\right] f(x)\right|^{p} \omega(x) d x\right)^{1 / p} \\
& \quad \leq\left(\int_{B\left(x_{0}, r\right)}\left|\left[b, T^{*}\right] f_{1}(x)\right|^{p} \omega(x) d x\right)^{1 / p}+\left(\int_{B\left(x_{0}, r\right)}\left|\left[b, T^{*}\right] f_{2}(x)\right|^{p} \omega(x) d x\right)^{1 / p} .
\end{aligned}
$$

By the $L_{\omega}^{p}$ boundedness of $\left[b, T^{*}\right]$ (see Theorem 2 in [8]), we obtain

$$
\int_{B\left(x_{0}, r\right)}\left|\left[b, T^{*}\right] f_{1}(x)\right|^{p} \omega(x) d x \leq C\left(1+\frac{r}{\rho\left(x_{0}\right)}\right)^{-\alpha} \omega\left(B\left(x_{0}, 2 r\right)\right)^{\lambda}\|f\|_{L_{\alpha, V, \omega}^{p, \lambda}\left(\mathbb{R}^{n}\right)}^{p} .
$$

Set $b_{B}=\frac{1}{\left|B\left(x_{0}, r\right)\right|} \int_{B\left(x_{0}, r\right)} b(x) d x$. Write $\left[b, T^{*}\right] f_{2}=\left(b-b_{B}\right) T^{*} f_{2}-T^{*}\left(f_{2}\left(b-b_{B}\right)\right)$. Then

$$
\begin{aligned}
& \left(\int_{B\left(x_{0}, r\right)}\left|\left[b, T^{*}\right] f_{2}(x)\right|^{p} \omega(x) d x\right)^{1 / p} \\
& \quad \leq\left(\int_{B\left(x_{0}, r\right)}\left|\left(b-b_{B}\right) T^{*} f_{2}\right|^{p} \omega(x) d x\right)^{1 / p}+\left(\int_{B\left(x_{0}, r\right)}\left|T^{*}\left(f_{2}\left(b-b_{B}\right)\right)\right|^{p} \omega(x) d x\right)^{1 / p} .
\end{aligned}
$$


By (8) in the proof of Theorem 1 , we obtain

$$
\begin{aligned}
& \int_{B\left(x_{0}, r\right)}\left|\left(b-b_{B}\right) T^{*} f_{2}\right|^{p} \omega(x) d x \\
& \quad \leq 2^{p-1}\left(\int_{B\left(x_{0}, r\right)}\left|b-b_{B}\right|^{p}\left(I_{1}(x)\right)^{p} \omega(x) d x+\int_{B\left(x_{0}, r\right)}\left|b-b_{B}\right|^{p}\left(I_{2}(x)\right)^{p} \omega(x) d x\right) .
\end{aligned}
$$

Let $p_{0}^{\prime} \leq p<\infty$. By simple computation, $\frac{p}{p_{0}^{\prime}}<1+\frac{p}{p^{\prime}}$. By Lemma $4, A_{p / p_{0}^{\prime}}^{\rho, \theta} \subseteq A_{1+p / p^{\prime}}^{\rho, \theta}$. Then

$$
\begin{aligned}
& \left(\frac{1}{\Psi\left(B\left(x_{0}, 2^{i} r\right)\right)\left|B\left(x_{0}, 2^{i} r\right)\right|} \int_{B\left(x_{0}, 2^{i} r\right)} \omega^{-p^{\prime} / p}(y) d y\right)^{p / p^{\prime}} \\
& \quad=\left(\frac{1}{\Psi\left(B\left(x_{0}, 2^{i} r\right)\right)\left|B\left(x_{0}, 2^{i} r\right)\right|} \int_{B\left(x_{0}, 2^{i} r\right)} \omega^{-\frac{1}{1+\frac{p}{p^{\prime}}-1}}(y) d y\right)^{\left(1+\frac{p}{p^{\prime}}-1\right)} \\
& \quad \leq C\left(\frac{1}{\Psi\left(B\left(x_{0}, 2^{i} r\right)\right)\left|B\left(x_{0}, 2^{i} r\right)\right|} \int_{B\left(x_{0}, 2^{i} r\right)} \omega(y) d y\right)^{-1} .
\end{aligned}
$$

By Lemma 1 and Corollary 2, as well as Lemma 3, we have

$$
\begin{aligned}
& \int_{B\left(x_{0}, r\right)}\left|b-b_{B}\right|^{p}\left(I_{1}(x)\right)^{p} \omega(x) d x \\
& \leq C_{N} \int_{B\left(x_{0}, r\right)}\left|b-b_{B}\right|^{p}\left(\int_{\left|x_{0}-z\right|>2 r} \frac{|f(z)|}{|x-z|^{n}\left(1+\frac{|x-z|}{\rho(x)}\right)^{N}} d z\right)^{p} \omega(x) d x \\
& \leq \sum_{i=1}^{\infty} C_{N} \int_{B\left(x_{0}, r\right)}\left|b-b_{B}\right|^{p}\left(\int_{B\left(x_{0}, 2^{i+1} r\right) \backslash B\left(x_{0}, 2^{i} r\right)} \frac{|f(z)|}{\left|x_{0}-z\right|^{n}\left(1+\frac{\left|x_{0}-z\right|}{\rho(x)}\right)^{N}} d z\right)^{p} \omega(x) d x \\
& \leq \sum_{i=1}^{\infty} C_{N} \int_{B\left(x_{0}, r\right)}\left|b-b_{B}\right|^{p} \frac{1}{\left(1+\frac{2^{i} r}{\rho(x)}\right)^{N p}}\left(2^{i} r\right)^{-n p}\left(\int_{B\left(x_{0}, 2^{i} r\right)}|f(z)| d z\right)^{p} \omega(x) d x \\
& \leq \sum_{i=1}^{\infty} C_{N} \int_{B\left(x_{0}, r\right)}\left|b-b_{B}\right|^{p} \frac{1}{\left(1+\frac{2^{i} r}{\rho(x)}\right)^{N p}}\left(2^{i} r\right)^{-n p} \\
& \times\left(\int_{B\left(x_{0}, 2^{i} r\right)}|f(z)| \omega(z)^{1 / p} \omega(z)^{-1 / p} d z\right)^{p} \omega(x) d x \\
& \leq \sum_{i=1}^{\infty} C_{N} \int_{B\left(x_{0}, r\right)}\left|b-b_{B}\right|^{p} \frac{1}{\left(1+\frac{2^{i} r}{\rho(x)}\right)^{N p}}\left(2^{i} r\right)^{-n p}\left(\int_{B\left(x_{0}, 2^{i} r\right)}|f(z)|^{p} \omega(z) d z\right) \\
& \times\left(\int_{B\left(x_{0}, 2^{i} r\right)} \omega(z)^{-\frac{p^{\prime}}{p}} d z\right)^{\frac{p}{p^{\prime}}} \omega(x) d x \\
& \leq \sum_{i=1}^{\infty} C_{N} \int_{B\left(x_{0}, r\right)}\left|b-b_{B}\right|^{p} \frac{1}{\left(1+\frac{2^{i} r}{\rho(x)}\right)^{N p}}\left(2^{i} r\right)^{-n p}\left(\int_{B\left(x_{0}, 2^{i} r\right)}|f(z)|^{p} \omega(z) d z\right) \\
& \times\left(1+\frac{2^{i} r}{\rho\left(x_{0}\right)}\right)^{\left(1+\frac{p}{p^{\prime}}\right) \theta}\left|B\left(x_{0}, 2^{i} r\right)\right|^{1+\frac{p}{p^{\prime}}} \omega\left(B\left(x_{0}, 2^{i} r\right)\right)^{-1} \omega(x) d x \\
& \leq \sum_{i=1}^{\infty} C_{N} \int_{B\left(x_{0}, r\right)}\left|b-b_{B}\right|^{p} \frac{\left(1+\frac{2^{i} r}{\rho\left(x_{0}\right)}\right)^{p \theta}}{\left(1+\frac{2^{i} r}{\rho(x)}\right)^{N p}} \omega\left(B\left(x_{0}, 2^{i} r\right)\right)^{-1}
\end{aligned}
$$




$$
\begin{aligned}
& \times \int_{B\left(x_{0}, 2^{i} r\right)}|f(z)|^{p} \omega(z) d z \omega(x) d x \\
\leq & \sum_{i=1}^{\infty} C_{N} \omega\left(B\left(x_{0}, 2^{i+1} r\right)\right)^{\lambda-1}\|f\|_{L_{\alpha, V, \omega}^{p, \lambda}\left(\mathbb{R}^{n}\right)}^{p} \int_{B\left(x_{0}, r\right)}\left|b-b_{B}\right|^{p} \frac{\left(1+\frac{2^{i} r}{\rho\left(x_{0}\right)}\right)^{-\alpha+p \theta}}{\left(1+\frac{2^{i} r}{\rho(x)}\right)^{N p}} \omega(x) d x \\
\leq & \sum_{i=1}^{\infty} C_{N} \omega\left(B\left(x_{0}, 2^{i+1} r\right)\right)^{\lambda-1} \omega\left(B\left(x_{0}, r\right)\right) \frac{\left(1+\frac{2^{i} r}{\rho\left(x_{0}\right)}\right)^{-\alpha+p \theta+\eta}}{\left(1+\frac{2^{i} r}{\rho\left(x_{0}\right)}\right)^{N p /\left(k_{0}+1\right)}}\|b\|_{B M O_{\rho}}^{p}\|f\|_{L_{\alpha, V, \omega}^{p, \lambda}}^{p}\left(\mathbb{R}^{n}\right) \\
\leq & \sum_{i=1}^{\infty} C_{N}\left(\frac{\omega\left(B\left(x_{0}, 2 r\right)\right)}{\omega\left(B\left(x_{0}, 2^{i+1} r\right)\right)}\right)^{1-\lambda} \omega\left(B\left(x_{0}, 2 r\right)\right)^{\lambda} \\
& \times \frac{\left(1+\frac{2^{i} r}{\rho\left(x_{0}\right)}\right)^{-\alpha+p \theta+\eta}}{\left(1+\frac{2^{i} r}{\rho\left(x_{0}\right)}\right)^{N p /\left(k_{0}+1\right)}\|b\|_{B M O_{\rho}}^{p}\|f\|_{L_{\alpha, V, \omega}^{p, \lambda}}^{p}\left(\mathbb{R}^{n}\right)} \\
\leq & \sum_{i=1}^{\infty} C_{N} 2^{-i n(1-\lambda) / q} \omega\left(B\left(x_{0}, 2 r\right)\right)^{\lambda} \frac{\left(1+\frac{2^{i} r}{\rho\left(x_{0}\right)}\right)^{-\alpha+p \theta+2 \eta}}{\left(1+\frac{2^{i} r}{\rho\left(x_{0}\right)}\right)^{N p /\left(k_{0}+1\right)}}\|b\|_{B M O_{\rho}}^{p}\|f\|_{L_{\alpha, V, \omega}^{p, \lambda}\left(\mathbb{R}^{n}\right)^{\prime}}^{p}
\end{aligned}
$$

where we choose $N$ large enough so that the above series converges.

For $I_{2}(x)$, we assume $n / 2<q<n$ due to Lemma 3. Then, since $x \in B\left(x_{0}, r\right)$, we also have $\frac{\left|x_{0}-z\right|}{2} \leq|x-z| \leq \frac{3\left|x_{0}-z\right|}{2}$. Then

$$
\begin{aligned}
& \int_{B\left(x_{0}, r\right)}\left|b-b_{B}\right|^{p}\left(I_{2}(x)\right)^{p} \omega(x) d x \\
& \leq C_{N} \int_{B\left(x_{0}, r\right)}\left|b-b_{B}\right|^{p}\left(\int_{\left|x_{0}-z\right|>2 r} \frac{|f(z)|}{|x-z|^{n-1}\left(1+\frac{|x-z|}{\rho(x)}\right)^{N}}\right. \\
&\left.\quad \times \int_{B(z,|x-z| / 4)} \frac{V(u)}{|u-z|^{n-1}} d u d z\right)^{p} \omega(x) d x \\
& \leq \sum_{i=1}^{\infty} C_{N} \int_{B\left(x_{0}, r\right)}\left|b-b_{B}\right|^{p}\left(\int_{B\left(x_{0}, 2^{i+1} r\right) \backslash B\left(x_{0}, 2^{i} r\right)} \frac{|f(z)|}{\left|x_{0}-z\right|^{n-1}\left(1+\frac{\left|x_{0}-z\right|}{\rho(x)}\right)^{N}}\right. \\
&\left.\quad \times \int_{B\left(x_{0}, 2^{i+3} r\right)} \frac{V(u)}{|u-z|^{n-1}} d u d z\right)^{p} \omega(x) d x \\
& \leq \sum_{i=1}^{\infty} C_{N} \int_{B\left(x_{0}, r\right)} \frac{1}{\left(1+\frac{2^{i} r}{\rho(x)}\right)^{N p}}\left(2^{i} r\right)^{-(n-1) p}\left|b-b_{B}\right|^{p} \\
& \quad \times\left(\int_{B\left(x_{0}, 2^{i} r\right)}|f(z)| \mathcal{I}_{1}\left(V \chi_{B\left(x_{0}, 2^{i} r\right)}\right) d z\right)^{p} \omega(x) d x .
\end{aligned}
$$

By (11) and (12) in the proof of Theorem 1, we obtain

$$
\begin{aligned}
& \int_{B\left(x_{0}, r\right)}\left|b-b_{B}\right|^{p}\left(I_{2}(x)\right)^{p} \omega(x) d x \\
& \leq C_{N} \int_{B\left(x_{0}, r\right)}\left|b-b_{B}\right|^{p}\left(\int_{\left|x_{0}-z\right|>2 r} \frac{|f(z)|}{|x-z|^{n-1}\left(1+\frac{|x-z|}{\rho(x)}\right)^{N}}\right. \\
& \left.\quad \times \int_{B(z,|x-z| / 4)} \frac{V(u)}{|u-z|^{n-1}} d u d z\right)^{p} \omega(x) d x
\end{aligned}
$$




$$
\begin{aligned}
& \leq \sum_{i=1}^{\infty} C_{N} \omega\left(B\left(x_{0}, 2^{i+1} r\right)\right)^{\lambda-1}\|f\|_{L_{\alpha, V, \omega}^{p, \lambda}}^{p}\left(\mathbb{R}^{n}\right) \\
& \times \int_{B\left(x_{0}, r\right)}\left|b-b_{B}\right|^{p} \frac{\left(1+\frac{2^{i} r}{\rho\left(x_{0}\right)}\right)^{-\alpha+(1+p / v) \theta+l_{0} p}}{\left(1+\frac{2^{i} r}{\rho(x)}\right)^{N p}} \omega(x) d x \\
& \leq \sum_{i=1}^{\infty} C_{N} \omega\left(B\left(x_{0}, 2^{i+1} r\right)\right)^{\lambda-1} \omega\left(B\left(x_{0}, r\right)\right) \\
& \times \frac{\left(1+\frac{2^{i} r}{\rho\left(x_{0}\right)}\right)^{-\alpha+(1+p / v) \theta+l_{0} p+\eta}}{\left(1+\frac{2^{i} r}{\rho\left(x_{0}\right)}\right)^{N p /\left(k_{0}+1\right)}}\|b\|_{B M O_{\rho}}^{p}\|f\|_{L_{\alpha, V, \omega}^{p, \lambda}}^{p}\left(\mathbb{R}^{n}\right) \\
& \leq \sum_{i=1}^{\infty} C_{N}\left(\frac{\omega\left(B\left(x_{0}, 2 r\right)\right)}{\omega\left(B\left(x_{0}, 2^{i+1} r\right)\right)}\right)^{1-\lambda} \omega\left(B\left(x_{0}, 2 r\right)\right)^{\lambda} \\
& \times \frac{\left(1+\frac{2^{i} r}{\rho\left(x_{0}\right)}\right)^{-\alpha+(1+p / v) \theta+l_{0} p+\eta}}{\left(1+\frac{2^{i} r}{\rho\left(x_{0}\right)}\right)^{N p /\left(k_{0}+1\right)}}\|b\|_{B M O_{\rho}}^{p}\|f\|_{L_{\alpha, V, \omega}^{p, \lambda}}^{p}\left(\mathbb{R}^{n}\right) \\
& \leq \sum_{i=1}^{\infty} C_{N} 2^{-i n(1-\lambda) / q} \omega\left(B\left(x_{0}, 2 r\right)\right)^{\lambda} \\
& \times \frac{\left(1+\frac{2^{i} r}{\rho\left(x_{0}\right)}\right)^{-\alpha+(1+p / v) \theta+l_{0} p+2 \eta}}{\left(1+\frac{2^{i} r}{\rho\left(x_{0}\right)}\right)^{N p /\left(k_{0}+1\right)}}\|b\|_{B M O_{\rho}}^{p}\|f\|_{L_{\alpha, V, \omega}^{p, \lambda}}^{p}\left(\mathbb{R}^{n}\right)
\end{aligned}
$$

if we choose $N$ large enough.

Now, for $x \in B\left(x_{0}, r\right)$ and using Lemma 3, we have

$$
\begin{aligned}
\left|T^{*}\left(f_{2}\left(b-b_{B}\right)\right)\right| & =\left|\int_{\left|x_{0}-z\right|>2 r} \mathcal{K}^{*}(x, z) f(z)\left(b-b_{B}\right) d z\right| \\
& \leq \tilde{I}_{1}(x)+\tilde{I}_{2}(x),
\end{aligned}
$$

where

$$
\tilde{I}_{1}(x)=C_{N} \int_{\left|x_{0}-z\right|>2 r} \frac{\left|f(z)\left(b-b_{B}\right)\right|}{|x-z|^{n}\left(1+\frac{|x-z|}{\rho(x)}\right)^{N}} d z
$$

and

$$
\tilde{I}_{2}(x)=C_{N} \int_{\left|x_{0}-z\right|>2 r} \frac{\left|f(z)\left(b-b_{B}\right)\right|}{|x-z|^{n-1}\left(1+\frac{|x-z|}{\rho(x)}\right)^{N}} \int_{B(z,|x-z| / 4)} \frac{V(u)}{|u-z|^{n-1}} d u d z
$$

Then,

$$
\begin{aligned}
& \left(\int_{B\left(x_{0}, r\right)}\left|T^{*}\left(f_{2}\left(b-b_{B}\right)\right)\right|^{p} \omega(x) d x\right)^{1 / p} \\
& \quad \leq\left(\int_{B\left(x_{0}, r\right)}\left(\tilde{I}_{1}(x)\right)^{p} \omega(x) d x\right)^{1 / p}+\left(\int_{B\left(x_{0}, r\right)}\left(\tilde{I}_{2}(x)\right)^{p} \omega(x) d x\right)^{1 / p} .
\end{aligned}
$$


Firstly, we consider $\tilde{I}_{1}(x)$. By Proposition 2 and (10), for $1 / p+1 / v+1 / s=1$, we have

$$
\begin{aligned}
& \int_{B\left(x_{0}, 2^{i} r\right)}\left|f(x)\left(b(x)-b_{B}\right)\right| d x \\
& \leq \int_{B\left(x_{0}, 2^{i} r\right)}|f(x)| \omega^{1 / p} \omega^{-1 / p}\left|b(x)-b_{B}\right| d x \\
& \leq \Psi\left(B\left(x_{0}, 2^{i} r\right)\right)\left|B\left(x_{0}, 2^{i} r\right)\right| \\
& \times\left(\frac{1}{\Psi\left(B\left(x_{0}, 2^{i} r\right)\right)\left|B\left(x_{0}, 2^{i} r\right)\right|} \int_{B\left(x_{0}, 2^{i} r\right)}|f(x)|^{p} \omega(x) d x\right)^{1 / p} \\
& \times\left(\frac{1}{\Psi\left(B\left(x_{0}, 2^{i} r\right)\right)\left|B\left(x_{0}, 2^{i} r\right)\right|} \int_{B\left(x_{0}, 2^{i} r\right)} \omega(x)^{-v / p} d x\right)^{1 / v} \\
& \times\left(\frac{1}{\Psi\left(B\left(x_{0}, 2^{i} r\right)\right)\left|B\left(x_{0}, 2^{i} r\right)\right|} \int_{B\left(x_{0}, 2^{i} r\right)}\left|b(x)-b_{B}\right|^{s} d x\right)^{1 / s} \\
& \leq C \Psi\left(B\left(x_{0}, 2^{i} r\right)\right)\left|B\left(x_{0}, 2^{i} r\right)\right| \\
& \times\left(\frac{1}{\Psi\left(B\left(x_{0}, 2^{i} r\right)\right)\left|B\left(x_{0}, 2^{i} r\right)\right|} \int_{B\left(x_{0}, 2^{i} r\right)}|f(x)|^{p} \omega(x) d x\right)^{1 / p} \\
& \times\left(\frac{1}{\Psi\left(B\left(x_{0}, 2^{i} r\right)\right)\left|B\left(x_{0}, 2^{i} r\right)\right|} \int_{B\left(x_{0}, 2^{i} r\right)} \omega(x) d x\right)^{-1 / p} \\
& \times\left(\frac{1}{\Psi\left(B\left(x_{0}, 2^{i} r\right)\right)\left|B\left(x_{0}, 2^{i} r\right)\right|} \int_{B\left(x_{0}, 2^{i} r\right)}\left|b(x)-b_{B}\right|^{s} d x\right)^{1 / s} \\
& \leq C\left(\Psi\left(B\left(x_{0}, 2^{i} r\right)\right)\right)^{1 / p+1 / v}\left|B\left(x_{0}, 2^{i} r\right)\right| \\
& \times\left(\int_{B\left(x_{0}, 2^{i} r\right)}|f(x)|^{p} \omega(x) d x\right)^{1 / p} \omega\left(B\left(x_{0}, 2^{i} r\right)\right)^{-1 / p} \\
& \times\left(\frac{1}{\left|B\left(x_{0}, 2^{i} r\right)\right|} \int_{B\left(x_{0}, 2^{i} r\right)}\left|b(x)-b_{B}\right|^{s} d x\right)^{1 / s} \\
& \leq C i\left(1+\frac{2^{i} r}{\rho\left(x_{0}\right)}\right)^{(1 / p+1 / v) \theta+\theta^{\prime}}\left|B\left(x_{0}, 2^{i} r\right)\right|\left(\int_{B\left(x_{0}, 2^{i} r\right)}|f(x)|^{p} \omega(x) d x\right)^{1 / p} \\
& \times \omega\left(B\left(x_{0}, 2^{i} r\right)\right)^{-1 / p}\|b\|_{B M O_{\rho}} \\
& \leq C i\left(1+\frac{2^{i} r}{\rho\left(x_{0}\right)}\right)^{(1 / p+1 / v) \theta+\theta^{\prime}}\left(2^{i} r\right)^{n}\left(\int_{B\left(x_{0}, 2^{i} r\right)}|f(x)|^{p} \omega(x) d x\right)^{1 / p} \\
& \times \omega\left(B\left(x_{0}, 2^{i} r\right)\right)^{-1 / p}\|b\|_{B M O_{\rho}} .
\end{aligned}
$$

Then we get

$$
\begin{aligned}
& \int_{B\left(x_{0}, r\right)}\left(\tilde{I}_{1}(x)\right)^{p} \omega(x) d x \\
& \quad=C_{N} \int_{B\left(x_{0}, r\right)}\left(\int_{\left|x_{0}-z\right|>2 r} \frac{\left|f(z)\left(b-b_{B}\right)\right|}{|x-z|^{n}\left(1+\frac{|x-z|}{\rho(x)}\right)^{N}} d z\right)^{p} \omega(x) d x \\
& \quad \leq \sum_{i=1}^{\infty} C_{N} \int_{B\left(x_{0}, r\right)}\left(\int_{B\left(x_{0}, 2^{i+1} r\right) \backslash B\left(x_{0}, i^{r} r\right)} \frac{\left|f(z)\left(b-b_{B}\right)\right|}{\left|x_{0}-z\right|^{n}\left(1+\frac{\left|x_{0}-z\right|}{\rho(x)}\right)^{N}} d z\right)^{p} \omega(x) d x
\end{aligned}
$$




$$
\begin{aligned}
& \leq \sum_{i=1}^{\infty} C_{N} \int_{B\left(x_{0}, r\right)} \frac{1}{\left(1+\frac{2^{i} r}{\rho(x)}\right)^{N p}}\left(2^{i} r\right)^{-n p}\left(\int_{B\left(x_{0}, 2^{i} r\right)}\left|f(z)\left(b-b_{B}\right)\right| d z\right)^{p} \omega(x) d x \\
& \leq \sum_{i=1}^{\infty} C_{N} i^{p} \int_{B\left(x_{0}, r\right)} \frac{\left(1+\frac{2^{i} r}{\rho\left(x_{0}\right)}\right)^{(1+p / v) \theta+p \theta^{\prime}}}{\left(1+\frac{2^{i} r}{\rho(x)}\right)^{N p}}\left(\int_{B\left(x_{0}, 2^{i} r\right)}|f(z)|^{p} \omega(z) d z\right) \\
& \times \omega\left(B\left(x_{0}, 2^{i} r\right)\right)^{-1}\|b\|_{B M O_{\rho}}^{p} \omega(x) d x \\
& \leq \sum_{i=1}^{\infty} C_{N} i^{p} \omega\left(B\left(x_{0}, 2^{i+1} r\right)\right)^{\lambda-1}\|f\|_{L_{\alpha, V, \omega}^{p, \lambda}\left(\mathbb{R}^{n}\right)}^{p}\|b\|_{B M O_{\rho}}^{p} \\
& \times \int_{B\left(x_{0}, r\right)} \frac{\left(1+\frac{2^{i} r}{\rho\left(x_{0}\right)}\right)^{-\alpha+(1+p / v) \theta+p \theta^{\prime}}}{\left(1+\frac{2^{i} r}{\rho(x)}\right)^{N p}} \omega(x) d x \\
& \leq \sum_{i=1}^{\infty} C_{N} i^{p} \omega\left(B\left(x_{0}, 2^{i+1} r\right)\right)^{\lambda-1} \omega\left(B\left(x_{0}, r\right)\right) \\
& \times \frac{\left(1+\frac{2^{i} r}{\rho\left(x_{0}\right)}\right)^{-\alpha+(1+p / v) \theta+p \theta^{\prime}}}{\left(1+\frac{2^{i} r}{\rho\left(x_{0}\right)}\right)^{N p /\left(k_{0}+1\right)}\|b\|_{B M O_{\rho}}^{p}\|f\|_{L_{\alpha, V, \omega}^{p, \lambda}}^{p}\left(\mathbb{R}^{n}\right)} \\
& \leq \sum_{i=1}^{\infty} C_{N} i^{p}\left(\frac{\omega\left(B\left(x_{0}, 2 r\right)\right)}{\omega\left(B\left(x_{0}, 2^{i+1} r\right)\right)}\right)^{1-\lambda} \omega\left(B\left(x_{0}, 2 r\right)\right)^{\lambda} \\
& \times \frac{\left(1+\frac{2^{i} r}{\rho\left(x_{0}\right)}\right)^{-\alpha+(1+p / v) \theta+p \theta^{\prime}}}{\left(1+\frac{2^{i} r}{\rho\left(x_{0}\right)}\right)^{N p /\left(k_{0}+1\right)}}\|b\|_{B M O_{\rho}}^{p}\|f\|_{L_{\alpha, V, \omega}^{p, \lambda}}^{p}\left(\mathbb{R}^{n}\right) \\
& \leq \sum_{i=1}^{\infty} C_{N} i^{p} 2^{-i n(1-\lambda) / q} \omega\left(B\left(x_{0}, 2 r\right)\right)^{\lambda}
\end{aligned}
$$

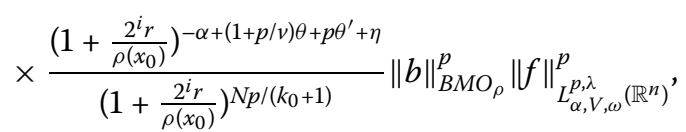

where we choose $N$ large enough so that the above series converges.

For $V \in B_{q}$, then $V \in B_{q+\varepsilon}$ for $\varepsilon>0$. Using Lemma 2, we get

$$
\begin{aligned}
\left\|V \chi_{B\left(x_{0}, 2^{i} r\right)}\right\|_{q+\varepsilon} & \leq C\left(2^{i} r\right)^{-n /(q+\varepsilon)^{\prime}} \int_{B\left(x_{0}, 2^{i} r\right)} V(x) d x \\
& \leq C\left(2^{i} r\right)^{-n /(q+\varepsilon)^{\prime}+n-2}\left(2^{i} r\right)^{-n+2} \int_{B\left(x_{0}, 2^{i} r\right)} V(x) d x \\
& \leq C\left(2^{i} r\right)^{-n /(q+\varepsilon)^{\prime}+n-2}\left(1+\frac{2^{i} r}{\rho\left(x_{0}\right)}\right)^{l_{0}} .
\end{aligned}
$$

Let $p_{0}^{\prime} \leq p<\infty$. We choose $u$ such that $u=\frac{q(q+\varepsilon)}{\varepsilon}$ and $1 / p+1 / v+1 / u+1 / s=1$. Let $1 /(q+\varepsilon)=1 / s+1 / n$. By simple computation,

$$
\begin{aligned}
\frac{p}{p_{0}^{\prime}} & =p\left(1-\frac{1}{q}+\frac{1}{n}\right)=p\left(1-\frac{1}{q}+\frac{1}{n}+\frac{1}{q+\varepsilon}-\frac{1}{q+\varepsilon}\right) \\
& =p\left(1-\frac{1}{q(q+\varepsilon)}-\frac{1}{s}\right)=1+\frac{p}{v}
\end{aligned}
$$


Lu and Wang Journal of Inequalities and Applications 2014, 2014:194

Page 14 of 16

http://www.journalofinequalitiesandapplications.com/content/2014/1/194

Finally, we deal with $\tilde{I}_{2}(x)$. Using Hölder's inequality, (10), and the boundedness of the fractional integral $\mathcal{I}_{1}: L^{q+\varepsilon} \rightarrow L^{s}$, for $1 / p+1 / v+1 / u+1 / s=1$, we have

$$
\begin{aligned}
& \int_{B\left(x_{0}, 2^{i} r\right)}\left|f(x)\left(b(x)-b_{B}\right)\right| \mathcal{I}_{1}\left(V \chi_{B\left(x_{0}, 2^{i} r\right)}\right) d x \\
& \leq \int_{B\left(x_{0}, 2^{i} r\right)}|f(x)| \omega^{1 / p} \omega^{-1 / p}\left|b(x)-b_{B}\right| \mathcal{I}_{1}\left(V \chi_{B\left(x_{0}, 2^{i} r\right)}\right) d x \\
& \leq\left(\Psi\left(B\left(x_{0}, 2^{i} r\right)\right)\right)^{1 / v}\left(\left|B\left(x_{0}, 2^{i} r\right)\right|\right)^{1 / v+1 / u}\left(\int_{B\left(x_{0}, 2^{i} r\right)}|f(x)|^{p} \omega(x) d x\right)^{1 / p} \\
& \times\left(\frac{1}{\Psi\left(B\left(x_{0}, 2^{i} r\right)\right)\left|B\left(x_{0}, 2^{i} r\right)\right|} \int_{B\left(x_{0}, 2^{i} r\right)} \omega(x)^{-v / p} d x\right)^{1 / v} \\
& \times\left(\frac{1}{\left|B\left(x_{0}, 2^{i} r\right)\right|} \int_{B\left(x_{0}, 2^{i} r\right)}\left|b(x)-b_{B}\right|^{u} d x\right)^{1 / u}\left(\int_{B\left(x_{0}, 2^{i} r\right)}\left(\mathcal{I}_{1}\left(V \chi_{B\left(x_{0}, 2^{i} r\right)}\right)\right)^{s} d x\right)^{1 / s} \\
& \leq C\left(\Psi\left(B\left(x_{0}, 2^{i} r\right)\right)\right)^{1 / v}\left(\left|B\left(x_{0}, 2^{i} r\right)\right|\right)^{1 / v+1 / u}\left(\int_{B\left(x_{0}, 2^{i} r\right)}|f(x)|^{p} \omega(x) d x\right)^{1 / p} \\
& \times\left(\frac{1}{\Psi\left(B\left(x_{0}, 2^{i} r\right)\right)\left|B\left(x_{0}, 2^{i} r\right)\right|} \int_{B\left(x_{0}, 2^{i} r\right)} \omega(x) d x\right)^{-1 / p} \\
& \times\left(\frac{1}{\left|B\left(x_{0}, 2^{i} r\right)\right|} \int_{B\left(x_{0}, 2^{i} r\right)}\left|b(x)-b_{B}\right|^{u} d x\right)^{1 / u}\left(\int_{B\left(x_{0}, 2^{i} r\right)}\left(\mathcal{I}_{1}\left(V \chi_{B\left(x_{0}, 2^{i} r\right)}\right)\right)^{s} d x\right)^{1 / s} \\
& \leq C\left(\Psi\left(B\left(x_{0}, 2^{i} r\right)\right)\right)^{1 / p+1 / v}\left|B\left(x_{0}, 2^{i} r\right)\right|^{1 / p+1 / v+1 / u} \\
& \times\left(\int_{B\left(x_{0}, 2^{i} r\right)}|f(x)|^{p} \omega(x) d x\right)^{1 / p} \omega\left(B\left(x_{0}, 2^{i} r\right)\right)^{-1 / p} \\
& \times\left(\frac{1}{\left|B\left(x_{0}, 2^{i} r\right)\right|} \int_{B\left(x_{0}, 2^{i} r\right)}\left|b(x)-b_{B}\right|^{u} d x\right)^{1 / u}\left\|\mathcal{I}_{1}\left(V \chi_{B\left(x_{0}, 2^{i} r\right)}\right)\right\|_{s} \\
& \leq C i\left(1+\frac{2^{i} r}{\rho\left(x_{0}\right)}\right)^{(1 / p+1 / v) \theta+\theta^{\prime}}\left|B\left(x_{0}, 2^{i} r\right)\right|^{1 / p+1 / v+1 / u}\left(\int_{B\left(x_{0}, 2^{i} r\right)}|f(x)|^{p} \omega(x) d x\right)^{1 / p} \\
& \times \omega\left(B\left(x_{0}, 2^{i} r\right)\right)^{-1 / p}\|b\|_{B M O_{\rho}}\left\|V \chi_{B\left(x_{0}, 2^{i} r\right)}\right\|_{q+\varepsilon} \\
& \leq C i\left(1+\frac{2^{i} r}{\rho\left(x_{0}\right)}\right)^{(1 / p+1 / v) \theta+\theta^{\prime}+l_{0}}\left(2^{i} r\right)^{(n-1)} \\
& \times \omega\left(B\left(x_{0}, 2^{i} r\right)\right)^{-1 / p}\left(\int_{B\left(x_{0}, 2^{i} r\right)}|f(x)|^{p} \omega(x) d x\right)^{1 / p}\|b\|_{B M O_{\rho}} .
\end{aligned}
$$

Then

$$
\begin{aligned}
& \int_{B\left(x_{0}, r\right)}\left(\tilde{I}_{2}(x)\right)^{p} \omega(x) d x \\
& \quad=C_{N} \int_{B\left(x_{0}, r\right)}\left(\int_{\left|x_{0}-z\right|>2 r} \frac{\left|f(z)\left(b-b_{B}\right)\right|}{|x-z|^{n-1}\left(1+\frac{|x-z|}{\rho(x)}\right)^{N}} \int_{B(z,|x-z| / 4)} \frac{V(u)}{|u-z|^{n-1}} d u d z\right)^{p} \omega(x) d x \\
& \quad \leq \sum_{i=1}^{\infty} C_{N} \int_{B\left(x_{0}, r\right)}\left(\int_{B\left(x_{0}, 2^{i+1} r\right) \backslash B\left(x_{0}, 2^{i} r\right)} \frac{\left|f(z)\left(b-b_{B}\right)\right|}{\left|x_{0}-z\right|^{n-1}\left(1+\frac{\left|x_{0}-z\right|}{\rho(x)}\right)^{N}}\right.
\end{aligned}
$$




$$
\begin{aligned}
& \left.\times \int_{B\left(x_{0}, 2^{i+3} r\right)} \frac{V(u)}{|u-z|^{n-1}} d u d z\right)^{p} \omega(x) d x \\
& \leq \sum_{i=1}^{\infty} C_{N} \int_{B\left(x_{0}, r\right)} \frac{1}{\left(1+\frac{2^{i} r}{\rho(x)}\right)^{N p}}\left(2^{i} r\right)^{-(n-1) p} \\
& \times\left(\int_{B\left(x_{0}, 2^{i} r\right)}\left|f(z)\left(b-b_{B}\right)\right| \mathcal{I}_{1}\left(V \chi_{B\left(x_{0}, 2^{i} r\right)}\right) d z\right)^{p} \omega(x) d x \\
& \leq \sum_{i=1}^{\infty} C_{N} i^{p} \omega\left(B\left(x_{0}, 2^{i+1} r\right)\right)^{\lambda-1}\|f\|_{L_{\alpha, V, \omega}^{p, \lambda}\left(\mathbb{R}^{n}\right)}^{p}\|b\|_{B M O_{\rho}}^{p} \\
& \times \int_{B\left(x_{0}, r\right)} \frac{\left(1+\frac{2^{i} r}{\rho\left(x_{0}\right)}\right)^{-\alpha+(1+p / v) \theta+p \theta^{\prime}+l_{0} p}}{\left(1+\frac{2^{i} r}{\rho(x)}\right)^{N p}} \omega(x) d x \\
& \leq \sum_{i=1}^{\infty} C_{N} i^{p} \omega\left(B\left(x_{0}, 2^{i+1} r\right)\right)^{\lambda-1} \omega\left(B\left(x_{0}, r\right)\right)
\end{aligned}
$$

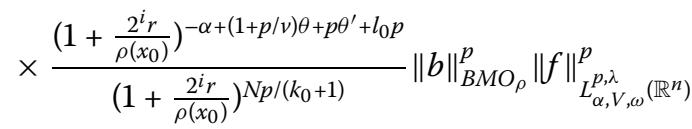

$$
\begin{aligned}
& \leq \sum_{i=1}^{\infty} C_{N} i^{p}\left(\frac{\omega\left(B\left(x_{0}, 2 r\right)\right)}{\omega\left(B\left(x_{0}, 2^{i+1} r\right)\right)}\right)^{1-\lambda} \omega\left(B\left(x_{0}, 2 r\right)\right)^{\lambda} \\
& \times \frac{\left(1+\frac{2^{i} r}{\rho\left(x_{0}\right)}\right)^{-\alpha+(1+p / v) \theta+p \theta^{\prime}+l_{0} p}}{\left(1+\frac{2^{i} r}{\rho\left(x_{0}\right)}\right)^{N p /\left(k_{0}+1\right)}}\|b\|_{B M O_{\rho}}^{p}\|f\|_{L_{\alpha, V, \omega}^{p, \lambda}\left(\mathbb{R}^{n}\right)}^{p} \\
& \leq \sum_{i=1}^{\infty} C_{N} i^{p} 2^{-i n(1-\lambda) / q} \omega\left(B\left(x_{0}, 2 r\right)\right)^{\lambda} \\
& \times \frac{\left(1+\frac{2^{i} r}{\rho\left(x_{0}\right)}\right)^{-\alpha+(1+p / v) \theta+p \theta^{\prime}+l_{0} p+\eta}}{\left(1+\frac{2^{i} r}{\rho\left(x_{0}\right)}\right)^{N p /\left(k_{0}+1\right)}}\|b\|_{B M O_{\rho}}^{p}\|f\|_{L_{\alpha, V, \omega}^{p, \lambda}}^{p},
\end{aligned}
$$

where we choose $N$ large enough so that the above series converges.

From (15)-(27), we obtain

$$
\left\|\left[b, T^{*}\right] f\right\|_{L_{\alpha, V, \omega}^{p, \lambda}\left(\mathbb{R}^{n)}\right.} \leq C\|f\|_{L_{\alpha, V, \omega}^{p, \lambda}\left(\mathbb{R}^{n}\right)} .
$$

Thus, we complete the proof of Theorem 2 .

\section{Competing interests}

The authors declare that they have no competing interests.

\section{Authors' contributions}

All authors contributed equally to the writing of this paper. All authors read and approved the final manuscript.

\section{Acknowledgements}

The authors would like to thank the referee for carefully reading which made the presentation more readable. This paper was supported by the National Natural Science Foundation of China under grant No. 10901018, the Fundamental Research Funds for the Central Universities, Program for New Century Excellent Talents in University and Beijing Natural Science Foundation under grant No. 1142005. 
References

1. Tang, L, Dong, JF: Boundedness for some Schrödinger type operators on Morrey spaces related to certain nonnegative potentials. J. Math. Anal. Appl. 355, 101-109 (2009)

2. Liu, Y, Wang, LJ: Boundedness for Riesz transform associated with Schrödinger operators and its commutator on Morrey spaces related to certain nonnegative potentials

3. Pan, GX, Tang, L: Boundedness for some Schrödinger type operators on weighted Morrey spaces. J. Funct. Spaces 2014, Article ID 878629 (2014)

4. Shen, ZW: $L^{p}$ Estimates for Schrödinger operators with certain potentials. Ann. Inst. Fourier (Grenoble) 45, 513-546 (1995)

5. Bongioanni, B, Harboure, E, Salinas, O: Analysis and assessment of ab initio three-dimensional prediction, secondary structure, and contacts prediction. J. Math. Anal. Appl. 373, 563-579 (2011)

6. Stein, EM: Harmonic Analysis: Real-Variable Methods, Orthogonality, and Oscillatory Integrals. Princeton University Press, Princeton (1993)

7. Tang, L: Weighted norm inequalities for Schrödinger type operators. Forum Math. (2013). doi:10.1515/forum-2013-0070

8. Bongioanni, B, Harboure, E, Salinas, O: Weighted inequalities for commutators of Schrödinger-Riesz transforms. J. Math. Anal. Appl. 392, 6-22 (2012)

9. Bongioanni, B, Harboure, E, Salinas, O: Commutators of Riesz transforms related to Schrödinger operators. J. Fourier Anal. Appl. 17, 115-134 (2011)

10.1186/1029-242X-2014-194

Cite this article as: Liu and Wang: Boundedness for Riesz transform associated with Schrödinger operators and its commutator on weighted Morrey spaces related to certain nonnegative potentials. Journal of Inequalities and Applications 2014, 2014:194

\section{Submit your manuscript to a SpringerOpen ${ }^{\ominus}$ journal and benefit from:}

- Convenient online submission

- Rigorous peer review

- Immediate publication on acceptance

- Open access: articles freely available online

- High visibility within the field

- Retaining the copyright to your article 